\title{
Recours ciblé à la sonde vésicale: plus rare, plus court, plus sûr!
}

\section{Stephanie Züllig}

Dr sc. nat., Directrice du programme pilote progress! La sécurité dans le sondage vésical, Sécurité des patients Suisse

\begin{tabular}{l}
\hline Références \\
Vous trouvez les références \\
de cet article sous \\
www.saez.ch $\rightarrow$ Numéro \\
actuel ou $\rightarrow$ Archives \\
$\rightarrow 2016 \rightarrow 46$ \\
\hline Correspondance: \\
Stephanie Züllig \\
Directrice du programme \\
pilote progress! La sécurité \\
dans le sondage vésical \\
Sécurité des patients Suisse \\
Asylstrasse 77 \\
CH-8032 Zurich \\
zuellig[at] \\
patientensicherheit.ch
\end{tabular}

Les sondes transurétrales à demeure (SAD) sont omniprésentes dans le quotidien médical. Bien que personne n'ignore qu'une sonde vésicale constitue un accès facile pour les bactéries et peut accroître le risque d'une bactériurie de 3 à 7\% par jour, le cathétérisme est appliqué à entre 20 et $25 \%$ de tous les patients hospitalisés. Parmi eux, un quart est touché par une infection des voies urinaires qui se développe en septicémie dans 3 à 5\% des cas. Environ la moitié des 350000 sondes vésicales posées chaque année en Suisse ne font pas l'objet d'une indication médicale claire.

Ces chiffres sont encore plus impressionnants si l'on tient compte du fait que la pose de la sonde va souvent de pair avec des complications telles que des traumatismes mécaniques des voies urinaires. Les conséquences des infections nosocomiales ou des blessures associées au cathétérisme ne sont pas anodines. Elles nécessitent un traitement, prolongent le séjour hospitalier et entraînent des coûts supplémentaires. Des études internationales montrent qu'avec des interventions spécifiques, il est possible de diminuer sensiblement la fréquence et la durée du sondage vésical. En parallèle, les risques d'infections et de lésions sont réduits d'autant.
La fondation Sécurité des patients a repris en Suisse cette thématique et elle a lancé, avec Swissnoso, le programme pilote national progress! La sécurité dans le sondage vésical. Il s'agit d'un programme de sensibilisation des professionnels et de mise en œuvre d'un faisceau d'interventions dans les hôpitaux suisses, visant à réduire le sondage vésical et les complications associées au cathétérisme. Les objectifs du projet sont:

- Plus rare: n'avoir recours au sondage vésical qu'en présence d'une liste claire d'indications

- Plus court: retirer la sonde vésicale le plus rapidement possible

- Plus sûr: assurer une pose et un suivi corrects des sondes vésicales

En vue d'atteindre ces objectifs, sept hôpitaux pilotes mettent en œuvre un faisceau d'interventions comportant les éléments suivants:

1. Liste des indications

La liste des indications se fonde sur des données probantes et spécifie les situations dans lesquelles le sondage vésical est justifié (voir encadré). Une liste négative en assure la délimitation. En fonction des possibilités, des solutions de rechange sont appliquées.

2. Contrôle quotidien des indications Un rappel indique qu'il faut contrôler le bien-fondé de l'indication. Si cette dernière n'est plus pertinente, la sonde doit être retirée le jour même.

3. Formation du personnel

Un cours de mise à jour permet de rafraîchir les connaissances relatives au mode de travail aseptique et l'application d'une technique de cathétérisme irréprochable. Cet entraînement est aussi l'occasion d'exercer ou de contrôler ses propres capacités.

La mise en œuvre qui débutera cet automne sera accompagnée par une campagne de sensibilisation. Grâce aux résultats des enquêtes d'évaluation, portant notamment sur le nombre de cathétérismes ou la fréquence des complications, le potentiel d'amélioration peut être estimé et l'efficacité des interventions attestée. La manière d'intégrer au mieux le faisceau d'interventions dans le quotidien clinique est décrite dans la recommandation de mesures: publication $n^{\circ} 9$, La sécurité dans le sondage vésical, disponible sur www.patientensicherheit.ch. 


\section{Références choisies:}

Magill SS, Edwards JR, Bamberg W, Beldavs ZG, Dumyati G, Kainer MA, et al. Multistate point-prevalence survey of health care-associated infections. N Engl J Med 2014 Mar 26;370(13):1198-208.

Saint S. Clinical and economic consequences of nosocomial catheter-related bacteriuria. Am J Infect Control 2000;28(1):68-75.

Gould CV, Umscheid CA, Agarwal RK, Kuntz G, Pegues DA, and the Healthcare Infection Control Practices Advisory Board (HICPAC). Guideline for Prevention of Catheter- Associated Urinary Tract Infections 2009. Infection Control and Hospital Epidemiology 2010 Apr 1;31(4):319-26.
Fakih MG, George C, Edson BS, Goeschel CA, Saint S. Implementing a national program to reduce catheter associated urinary tract infection: a quality improvement collaboration of state hospital associations, academic medical centers, professional societies, and governmental agencies. Infect Control Hosp Epidemiol 2013 Oct;34(10):1048-54.

Meddings J, Saint S, Fowler KE, Gaies E, Hickner A, Krein SL, et al. The Ann Arbor Criteria for Appropriate Urinary Catheter Use in Hospitalized Medical Patients: Results Obtained by Using the RAND/UCLA Appropriateness Method. Ann Intern Med 2015 May 5;162(9 Supplement):S1-S34. 